\title{
Visualisation of natural aquatic colloids and particles - a comparison of conventional high vacuum and environmental scanning electron microscopy
}

\author{
Frederic J. Doucet, $\uparrow^{a}$ Jamie R. Lead, ${ }^{* a}$ Leanne Maguire, ${ }^{a}$ Eric P. Achterberg $\ddagger^{b}$ and \\ Geoff E. Millward ${ }^{b}$
}
${ }^{a}$ Division of Environmental Health and Risk Management, School of Geography, Earth and Environmental Sciences, University of Birmingham, Edgbaston, Birmingham, UK B15 2TT. E-mail: j.r.lead@bham.ac.uk
${ }^{b}$ School of Earth, Ocean and Environmental Sciences, University of Plymouth, Plymouth, UK PL4 8AA

Received 10th September 2004, Accepted 15th December 2004

First published as an Advance Article on the web 11th January 2005

\begin{abstract}
The applicability of environmental scanning electron microscopy (ESEM; imaging of hydrated samples) and conventional high vacuum scanning electron microscopy (SEM; imaging of dried samples at high vacuum) for the observation of natural aquatic colloids and particles was explored and compared. Specific attention was given to the advantages and limitations of these two techniques when used to assess the sizes and morphologies of complex and heterogeneous environmental systems. The observation of specimens using SEM involved drying and coating, whereas ESEM permitted their examination in hydrated form without prior sample preparation or conductive coating. The two techniques provided significantly different micrographs of the same sample. SEM provided sharper images, lower resolution limits (10 $\mathrm{nm}$ or lower), but more densely packed particles, suggesting aggregation, and different morphological features than ESEM, suggesting artefacts due to drying. ESEM produced less easily visualised materials, more complex interpretation, slightly higher resolution limits $(30-50 \mathrm{~nm})$, but these limitations were more than compensated for by the fact that ESEM samples retained, at least to some extent, their morphological integrity. The results in this paper show that SEM and ESEM should be regarded as complementary techniques for the study of aquatic colloids and particles and that ESEM should be more widely applied to aquatic environmental systems than hitherto.
\end{abstract}

\section{Introduction}

Natural aquatic colloids and particles are defined as materials with sizes ranging between $1 \mathrm{~nm}$ and $1 \mu \mathrm{m},{ }^{1}$ and greater than 1 $\mu \mathrm{m}$, respectively. They are composed of inorganic oxides of aluminium, iron, manganese and silicon, organic humic substances and polysaccharides, as well as carbonates, clays and microbes including viruses and bacteria. These individual components are generally intimately associated with each other to form complex mixtures. ${ }^{2,3}$ It is now widely acknowledged that these materials play a significant role in controlling the speciation of essential nutrients and trace metals and in affecting metal transport and metal biological availability and toxicity in the aquatic environment. ${ }^{4}$ Despite a large number of studies that have focused on elucidating the structure and role of colloids and particles in natural waters, these ubiquitous components have successfully retained most of their mystery. This is essentially due to their presence at low concentrations and to their delicate structures and conformation, which can easily be denatured by sampling and sample handling. This difficulty has necessitated the development of minimally perturbing techniques for their fractionation and visualisation and for the quantitative analysis of their properties. ${ }^{5}$ In addition, it is essential to use a range of techniques to fully appreciate the complex structure of natural aquatic colloids and particles. ${ }^{6-8}$

$\dagger$ Present address: Division of Food Sciences, School of Biosciences, University of Nottingham, Sutton Bonington Campus, Loughborough, UK LE12 5RD.

$\ddagger$ Present address: Southampton Oceanography Centre, School of Ocean and Earth Science, University of Southampton, European Way, Southampton, UK SO14 3ZH
Examples of powerful visualisation techniques that have been commonly used in recent years are atomic force microscopy $(\mathrm{AFM})^{3,9-12}$ and transmission electron microscopy (TEM). ${ }^{3,13,14}$

Recently, advances in microscopy have been made, with the advent of environmental scanning electron microscopy (ESEM), for the visualisation of hydrated specimens at relatively high resolution. ${ }^{15}$ This technique has now found a number of applications, such as (i) the exploration of adhesive materials from marine organisms ${ }^{16}$ (ii) the investigation of the role of microorganisms in degradation processes, ${ }^{17,18}$ (iii) the observation of structural changes of the intestinal mucosa of rats, ${ }^{19}$ (iv) the imaging of dynamic processes such as dehydration and rehydration, ${ }^{20-23}(\mathrm{v})$ the observation of the microstructure of hydrated bacterial biofilms and associated extracellular polymeric substances, ${ }^{24,25}$ (vi) the structural study of bacteriogenic sulfur deposits ${ }^{26}$ and (vii) the examination of biomaterials. ${ }^{27}$ However, comparatively little work has yet been done applying this potentially powerful tool for the investigation of natural aquatic colloids and particles. To the best of our knowledge, only four studies have attempted to provide an insight into colloidal morphologies using ESEM. Nutall and Kale ${ }^{28}$ examined the structure of natural groundwater colloids captured by a laboratory-scale packed column, and Lavoie et al., ${ }^{21}$ studied large marine aggregates through different stages of hydration, from completely immersed in water to partially dessicated. ESEM has also been used to monitor the structures of humic substances as a function of relative humidity and $\mathrm{pH},{ }^{29}$ which demonstrated that dehydration of organic structures had promoted their aggregation, which strongly supports the case that the drying step preceding 
SEM observation of natural colloids and particles affects, perhaps irreversibly, their conformation. In addition, we recently reported the applicability of ESEM, and other microscopy techniques, to assess the ability of cross-flow filtration (CFF) to perform adequate size fractionation of freshwater colloids and particles. ${ }^{12}$

The ESEM operating system has been detailed elsewhere en-32 $^{30}$ and progress made in the development of the technique has been reviewed recently. ${ }^{33,34}$ The benefits of using ESEM are accomplished by eliminating the high vacuum requirements of scanning electron microscopy (SEM) in the microscope sample chamber. The instrument allows the visualisation of specimens in a saturated water vapour environment, thus permitting the study of fully hydrated samples maintained in their native state. The acceleration voltage often used in ESEM is lower than that typically used in SEM imaging, which permits lessdestructive imaging of fragile specimens. Sample handling and preparation are also kept minimal, reducing the risk of altering their structure. In addition, ESEM does not require coating of the samples with a conducting layer. ${ }^{32}$ It is capable of a resolution limit of $c a .30 \mathrm{~nm}$ at a relative humidity as high as $65 \%,{ }^{29}$ which is slightly higher than with SEM $(\mathrm{ca} .1 \mathrm{~nm}) .{ }^{20}$ Finally, imaging hydrated samples using ESEM does not require poorly electron dense materials such as humic substances to be stained. In contrast to ESEM, imaging with a conventional high vacuum SEM requires drying and coating of wet samples, and staining of poorly electron dense materials, which may cause significant structural disruption of the samples. Therefore, in principle, ESEM imaging would appear to be the method of choice for imaging natural aquatic colloidal and particulate dispersions with minimal disturbance. The fact that scarce attention has been devoted to the applicability of ESEM to aquatic colloidal and particulate systems is therefore surprising, although the technique is relatively new and expensive.

In the present work, the comparison of SEM and ESEM was undertaken to ascertain some of their advantages, limitations and applicability in studying the sizes and morphologies of natural aquatic colloids and particles.

\section{Experimental procedures}

\section{Sampling and size fractionation}

Surface water samples were collected in translucent high density polyethylene drums (20 1; Fisher UK Ltd) from the Tamar Estuary, Southwest England, in September 2003, at a salinity of less than 0.5 units. Sampling was performed from the bank and water was collected just below the surface. All containers were cleaned in $10 \%$ nitric acid solution for $24 \mathrm{~h}$, and thoroughly rinsed with ultra-pure water prior to their use. A final rinse was performed with the estuarine water and the washings were discarded. Colloidal and particulate separation of the water into two nominal size fractions was performed using a commercial Millipore Pellicon 2 bench-top cross-flow filtration device (Millipore UK Ltd) following a $12 \mathrm{~h}$ settling period after sampling. The bulk water from the Tamar was fractionated using a Durapore ${ }^{\mathrm{TM}}$ polyvinylidene fluoride cassette filter $(0.45 \mu \mathrm{m}$ pore size $)$ with a surface area of $0.5 \mathrm{~m}$, at a concentration factor of $c a .4$ (defined as the ratio of the feed flow rate to the retentate flow rate). This resulted in the generation of a permeate (i.e. the fraction passing through the CFF membrane) and a retentate (i.e. the fraction retained by the membrane). Prior to CFF fractionation, the membranes were pre-conditioned with 101 of the corresponding samples. Upon completion of the separation, the colloidal and particulate fractions were refrigerated at $4{ }^{\circ} \mathrm{C}$ and stored in the dark in polyethylene bottles (pre-cleaned as above). All analyses were performed within $3 \mathrm{~d}$ following sampling and fractionation.

\section{SEM imaging}

Images of the natural and the CFF-generated fractions were collected using ESEM and high vacuum SEM. A Philips XL 30 field emission gun (FEG) environmental scanning electron microscope was used for all experiments.

ESEM. This technique was used to observe the morphology of hydrated colloidal and particulate fractions in their native, hydrated state. Droplets of the Tamar and CFF-generated samples were placed directly onto clean glass surfaces, which were fixed to ESEM stainless steel support stubs using silver paint in order to reduce charge effects around the sample. These were in turn positioned on a Peltier effect cooling stage. Four drops of ultra-pure water were placed on the cooling stage around the sample to minimise or inhibit any dehydration that may occur during evacuation of the air from the sample chamber. The pressure in the chamber was then reduced to 6 Torr. Saturation of the sample environment with water was ensured by flooding the chamber with water vapour several times to 10 Torr. Finally, water pressure was slowly dropped to the imaging pressure (see below). The temperature of the stage was maintained at $2{ }^{\circ} \mathrm{C}$ at all times. Microscopy observations were performed with a standard solid-state gaseous secondary electron detector using a low acceleration voltage of $10 \mathrm{kV}$ (to reduce beam damage) at a spot size of 4.0. Imaging was performed at a chamber pressure ranging from 2.0 (i.e. relative humidity of $c a .40 \%$ ) to 3.9 (i.e. relative humidity of $c a .75 \%$ ) Torr. Particle size distributions (PSD) were also estimated by measuring the lateral dimensions of around 250 single particles.

SEM. The same electron microscope was also used in high vacuum mode to obtain information on morphologies and size distribution of the same waters and CFF fractions. Droplets of each sample were placed onto a clean electron microscopy support stub, allowed to air dry and sputter-coated with platinum in an Emscope SC500. The acceleration voltage was $15 \mathrm{kV}$. PSD were also determined by measuring the lateral dimension of around 250 single particles.

\section{Results and discussion}

Fig. 1 illustrates typical SEM (left column) and ESEM (right column) micrographs of the Tamar estuary and the CFFgenerated fractions. The two techniques provided very different images for all fractions. The most obvious difference was observed for the permeate, where the SEM image (Fig. 1G) showed full coverage of the surface of the stub by at least one layer of densely packed particles, whereas ESEM (Fig. 1H) provided partial coverage of the stub by a mixture of individual particles and small aggregates. This was the first indication that the distinctive sample preparation methods and imaging procedures characteristic to each technique had affected the samples in different ways.

\section{SEM imaging and artefacts}

SEM provided very clear images of dried and coated samples. Analysis of the images revealed a number of distinct particle morphologies (Figs. 1 and 2). Irregularly shaped material (but often very roughly spherical) was the most dominant feature. Rod-shaped (Figs. 1E and 2D) and larger and more regularly spherical materials (Figs. 1A, 1C, 1E, 2D and 2E), presumably biological cells and debris primarily of diatom origin, were also observed. Flat lemon-shaped particles observed in Fig. 2B were comparable to materials reported in another work $\mathrm{w}^{2,20}$ and were also likely to be diatom remains. The oval-shaped particles in Fig. 2C were presumably some forms of relatively well preserved bacteria, based on their size and shape. An image of a 

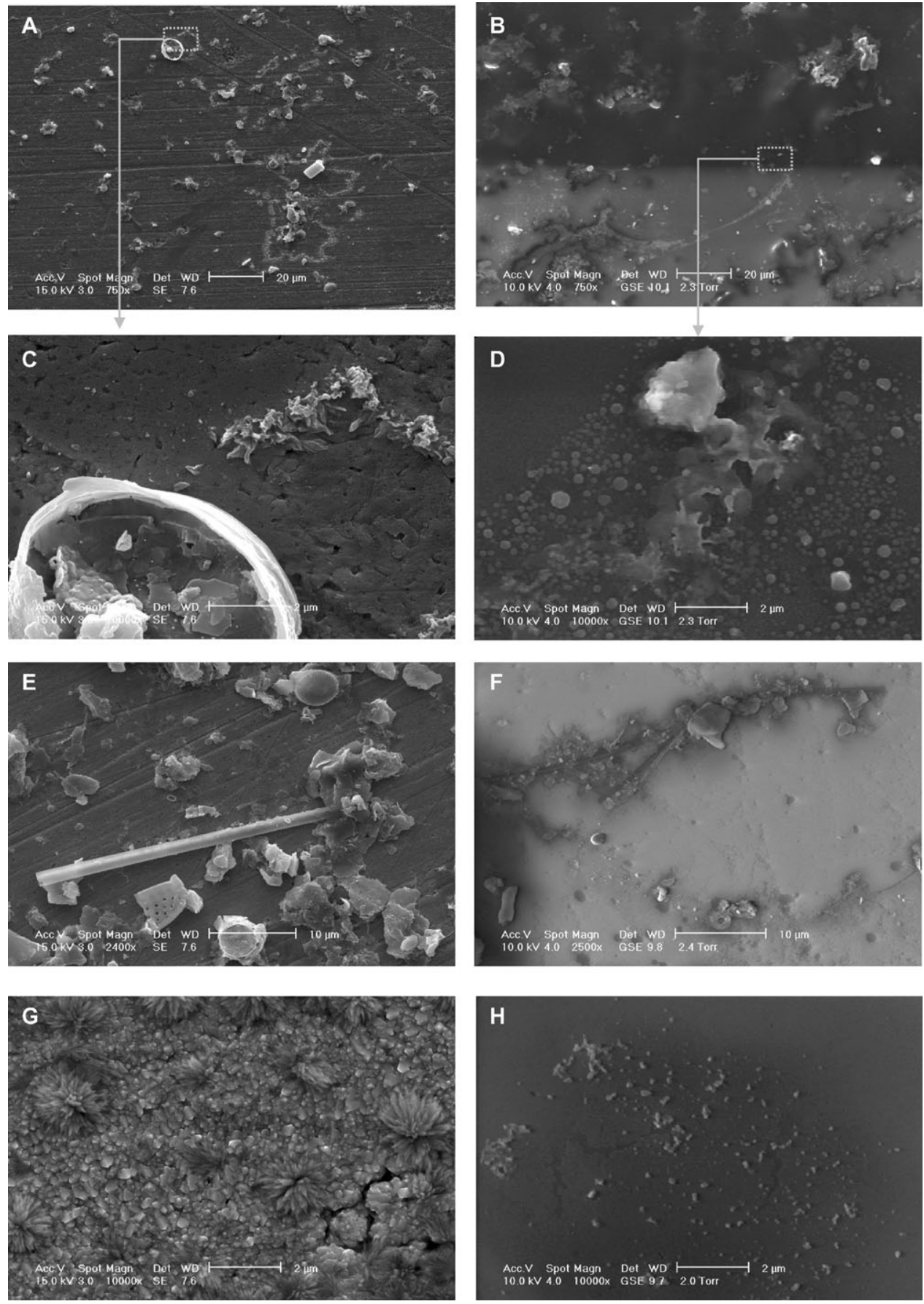

Fig. 1 Comparison between SEM and ESEM micrographs of estuarine samples, pre- and post-fractionation (SEM: A. unfractionated Tamar sample, C. high magnification version of A., E. retentate, G. permeate; ESEM: B. unfractionated Tamar estuary, D. high magnification version of B., F. retentate, H. permeate).

freshly clean stub is also shown (Fig. 2A) to help the reader to differentiate between the stub surface and the samples. The stub surface was characterised by an unsmoothed surface with distinct stripes up to a few $\mu \mathrm{m}$ wide.

At least two types of artefacts were identified. First, Fig. 1G and to a lesser extent Fig. 1A and 1C illustrate the presence of fairly crystalline structures. Since the samples used in this study originated from estuarine waters, it is conceivable that the drying process promoted areas with localised excess of salt, and that subsequent salt crystal nucleation occurred for which small natural colloids and particles or defects in the stub surface may have acted as templates. Second, for the same permeate sample, Fig. 1G exhibits densely-packed particles which were not featured using ESEM (Fig. 1H). This suggested that aggregation had occurred and the SEM images may not be fully realistic.

\section{ESEM imaging and artefacts}

At $100 \%$ relative humidity (i.e. water pressure of 5.4 Torr) in ESEM, the hydrated samples were fully covered by a layer of free water, which made colloid visualisation impossible. No exploitable images could therefore be generated under this condition. The water overlying the samples was gradually removed by slowly reducing the vapour pressure until the colloids became visible. The maximum relative humidity at which usable images could be recorded was found to be $c a$. $75 \%$ (i.e. water pressure of 4.0 Torr), from which point the samples came into relief and focusing became possible. Decreasing the relative humidity even further must be performed very carefully since partial drying of the samples often induces their shrinking (e.g. Mn oxyhydroxides) ${ }^{35}$ or their aggregation (e.g. humic substances), ${ }^{29}$ depending on the surface properties 

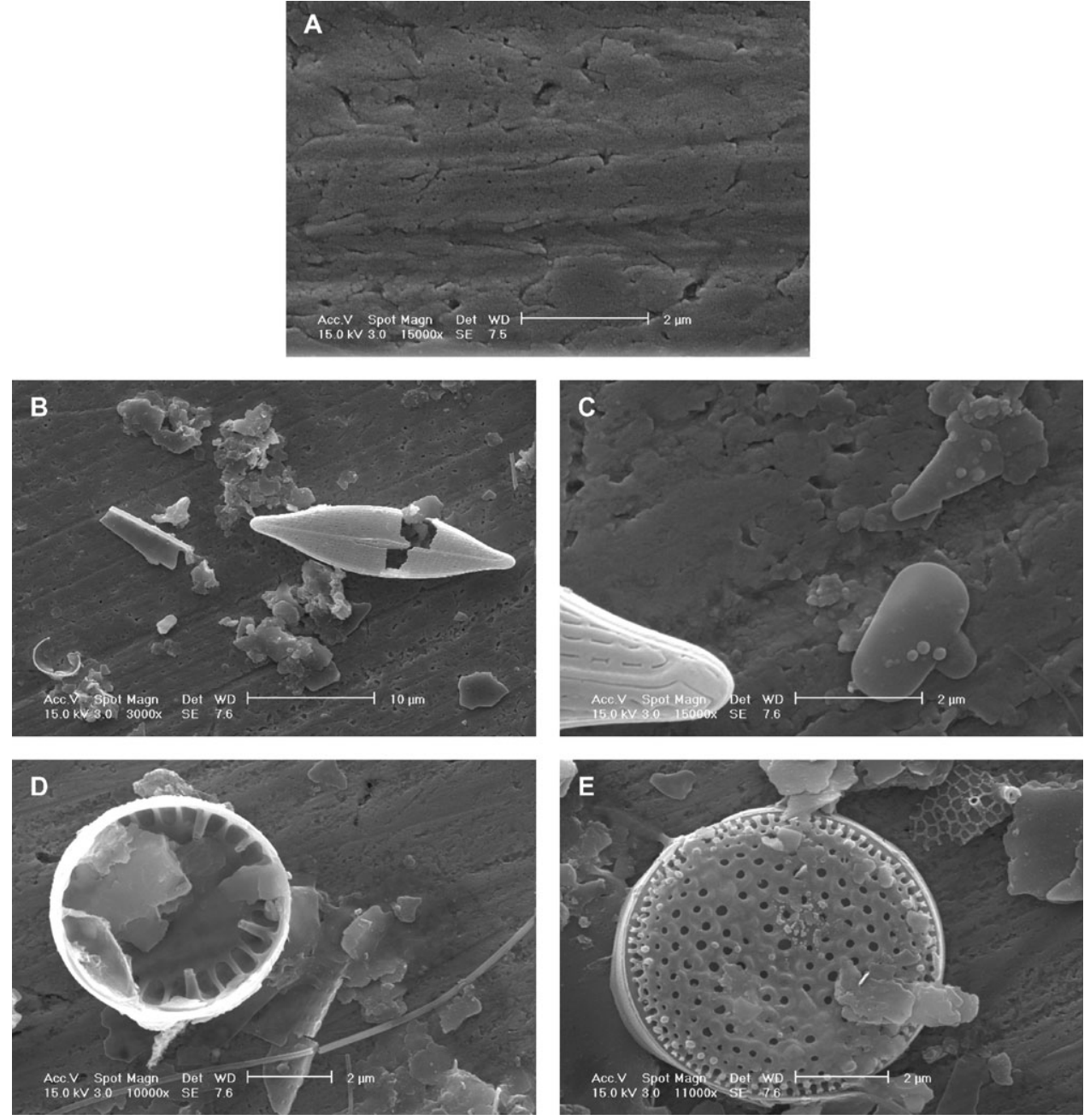

Fig. 2 Examples of SEM recorded micrographs of a clean stub surface (A.) and particles of varying morphologies (B. to E.)

and conformation of the materials under observation. This means that particle size can be significantly affected and may not be fully representative of the sample composition. ${ }^{12}$
Not all morphologies identified by SEM were seen using ESEM. For instance, materials such as diatoms and rod-shaped particles were not observed, perhaps due to coverage by free
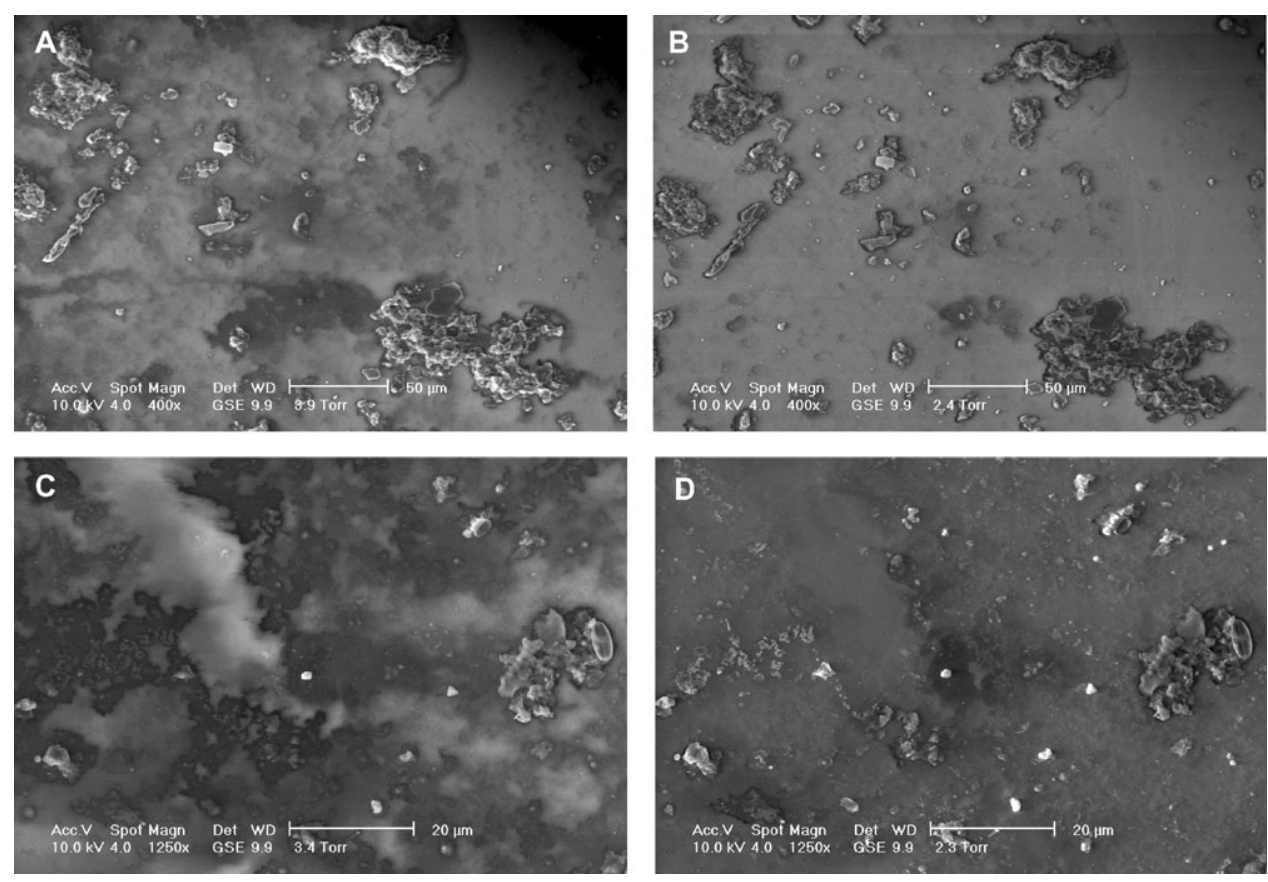

Fig. 3 ESEM micrographs recorded at different water vapour pressure (unfractionated Tamar sample) at 3.9 Torr (A.) and 2.4 Torr (B.); Retentate at 3.4 Torr (C.) and 2.3 Torr (D.). 

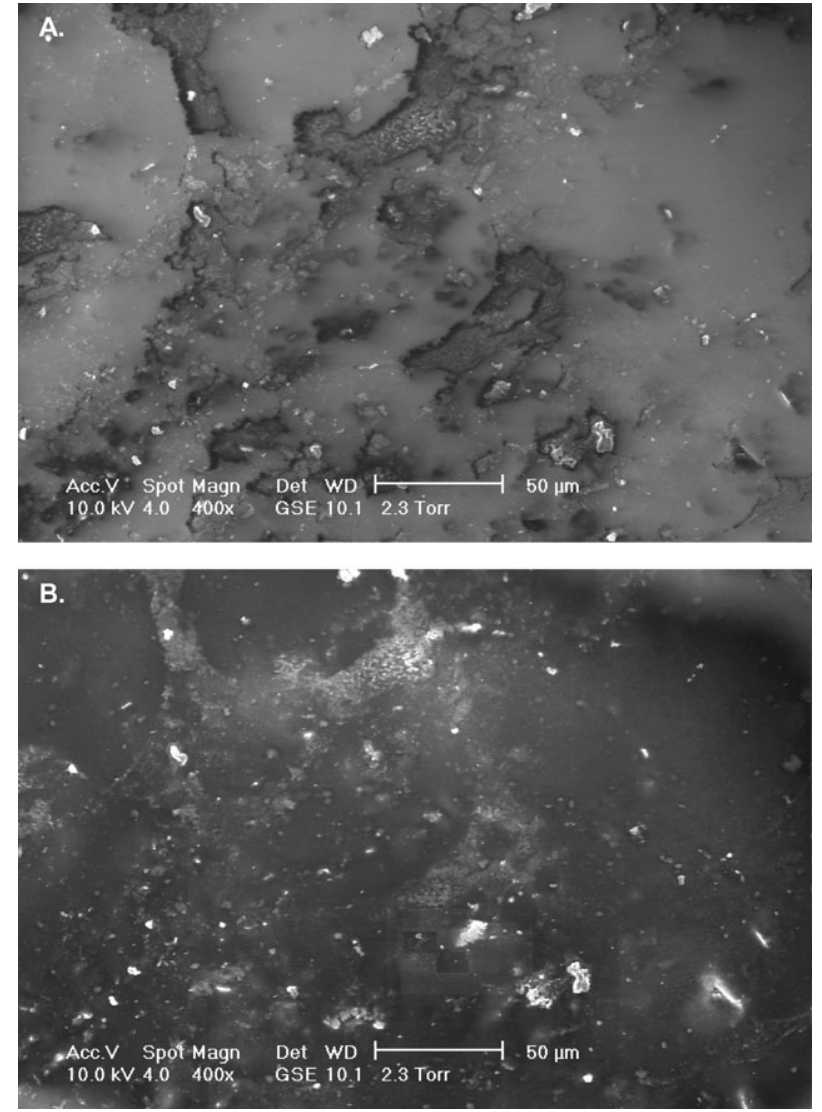

Fig. 4 ESEM micrographs of unfractionated Tamar sample recorded at a water vapour pressure of 2.3 Torr after $1 \mathrm{~min}$ (A.) and $6 \mathrm{~min}$ (B.).

water. However, many featureless, swollen gel-like structures were imaged (Figs. 1B and 1D, zoomed in from Fig. 1B).

In contrast to SEM, no salt crystallisation was observed during ESEM imaging when working at vapour pressure as low as 2 Torr, although such artefacts have already been reported for marine samples. ${ }^{16,21}$ Presumably, the salts in estuarine waters may be less of a problem than in marine samples for the visualisation of natural materials using ESEM.
Another study that quantified the structures of standard Suwannee River humic acids using fractal dimensions ${ }^{29}$ also observed the formation of large crystals of $\mathrm{NaCl}$ during dehydration of their $\mathrm{NaCl}$-based sample matrix in ESEM, although those appeared differently shaped than in this study.

An ESEM generated artefact is noticeably shown in Fig. 1D. A large number of fairly spherical features in the size range 0.1 $-0.6 \mu \mathrm{m}$ were clearly visible, and were very similar to previously published observations. ${ }^{16}$ These are presumably protruding droplets of water that condensed onto the stub surface during dehydration. Micrographs that were recorded at relative humidity as low as 2 Torr showed that at all time the surface of the stub was covered with free water (Figs. 1B, 1D, $1 \mathrm{~F}$ and $1 \mathrm{H})$. This made the focussing in ESEM challenging, as discussed later.

The image acquisition at water pressures between 3 and 4 Torr was still challenging due to the amount of free water covering a large proportion of particles (Fig. 3). Reducing the pressure to about 2.3 Torr helped to make the particles more visible, to better define the edges of particles and to significantly improve the image quality as a whole. However, care must be taken not to drastically change the nature of the samples. The present work showed no evidence that changes in the sample structure or aggregation had taken place, but Redwood et al. ${ }^{29}$ demonstrated that material such as humic substances, the major organic constituents of soils and aquatic environments, ${ }^{36,37}$ tend to aggregate when subjected to dehydration. Fig. 4 shows two micrographs of the same sample taken at the same water pressure (2.3 Torr) but 5 min apart. The second micrograph (Fig. 4B) shows a large number of particles that were not visible in Fig. 4A, due to their coverage by free water. This demonstrated that the removal of free water had occurred gradually over at least several minutes and immediate imaging may not be advisable. It is essential to account for this and ensure imaging conditions are identical between different samples.

In principle, imaging wet specimens by ESEM at a resolution as low as $10 \mathrm{~nm}$ is possible under ideal conditions (low relative humidity, well defined surfaces) whereas lower spatial resolutions are possible at higher relative humidity due to the vapour skirt. ${ }^{32}$ Regularly shaped humic substances can be viewed at a resolution of $c a .30 \mathrm{~nm}$ at a relative humidity as high as $65 \%$
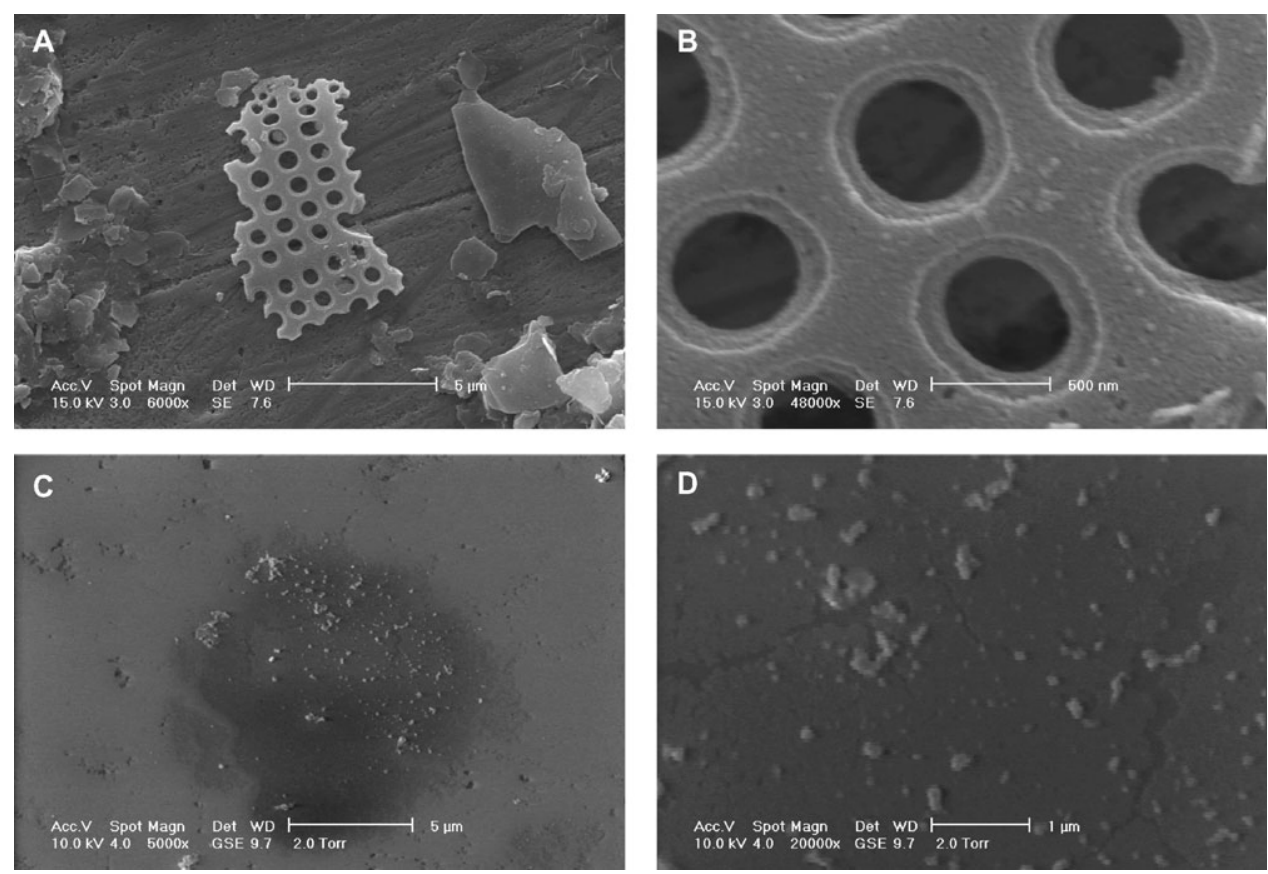

Fig. 5 Micrographs illustrating the resolution limits achievable with SEM and ESEM (A. (low magnification) and B. (high magnification) SEM; C. (low magnification) and D. (high magnification) ESEM). 
(a)
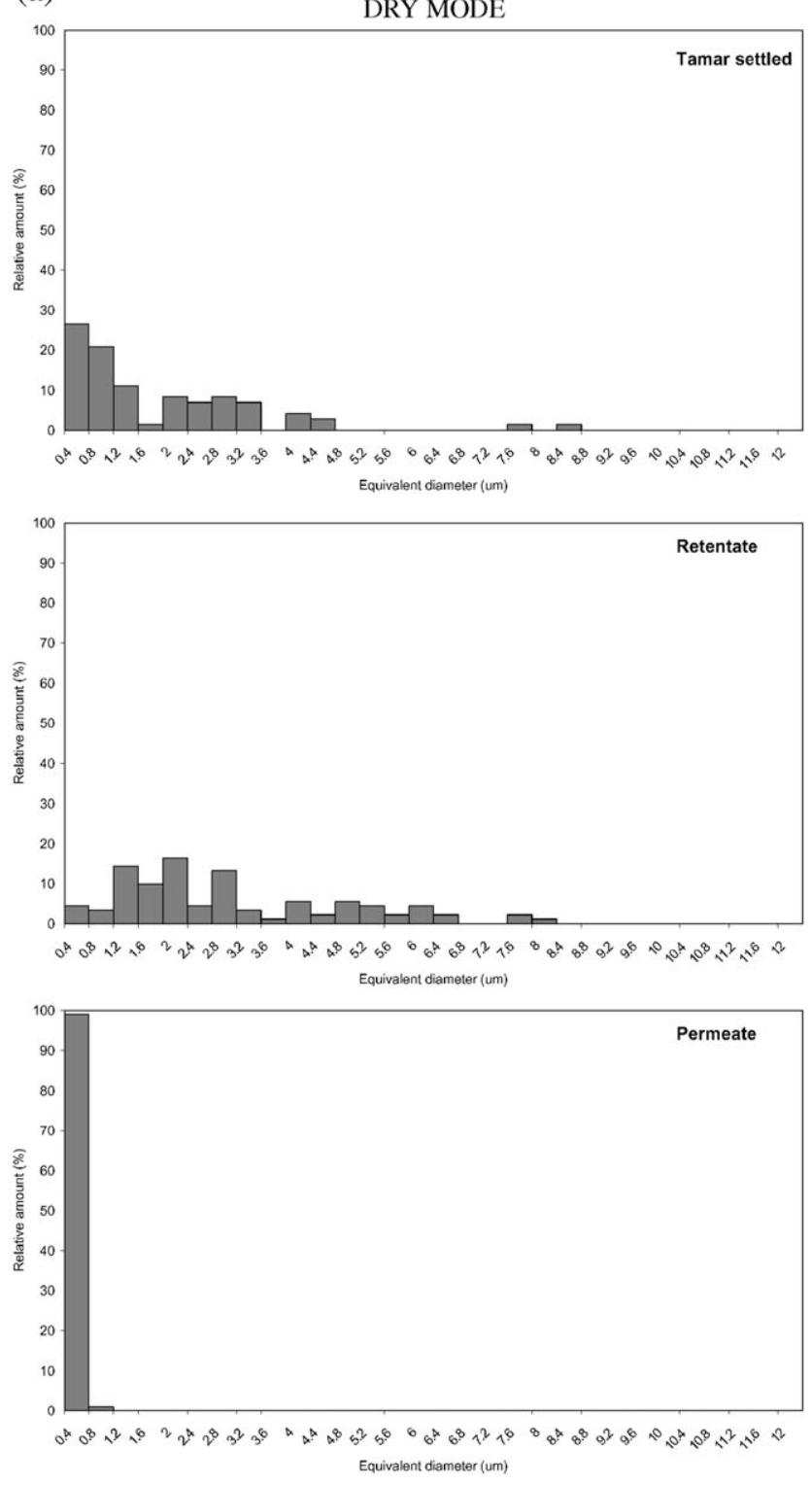

(b)

WET MODE
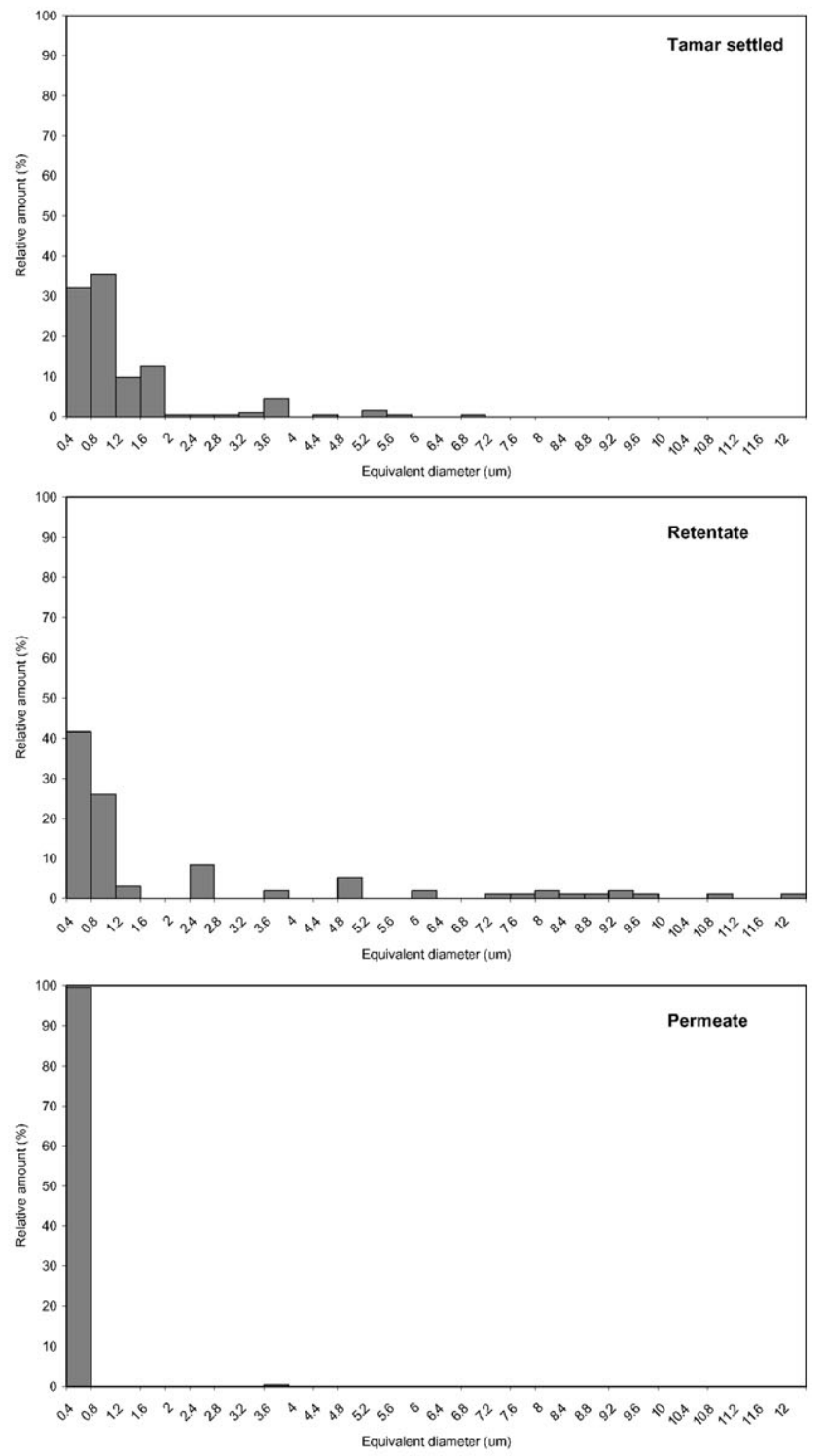

Fig. 6 Size distribution histograms from SEM (A.) and ESEM (B.) analysis (CFF fractionation at CF of ca. 4; number of particles measured: 250).

although imaging must be performed very carefully. ${ }^{29}$ However, many environmental particles are relatively amorphous and gel-like and our experience in this work suggests that a resolution limit of about $50 \mathrm{~nm}$ is realistic here (Fig. 5D, which is a highly magnified version of Fig. 5C). In contrast, SEM permitted a resolution limit of about $10 \mathrm{~nm}$ or lower to be achieved (Fig. 5B, which is a magnified version of Fig. 5A, presumably showing part of a diatom).

\section{Particle size distribution: SEM vs. ESEM}

Size distributions of deposited material were also quantified based on the SEM and ESEM images (Figs. 6A and 6B respectively) and were also technique-dependent. For instance, analysis of the images of the unfractionated Tamar sample revealed that less than $10 \%$ of the material imaged by ESEM were $>2 \mu \mathrm{m}$ whereas SEM showed about $32 \%$ of the material $>2 \mu \mathrm{m}$. Although the size distributions extracted from the SEM and ESEM images of the permeate were in agreement, it was not the case for the retentate, where the amount of material $<0.45 \mu \mathrm{m}$ found by SEM $(<5 \%)$ and ESEM $(>40 \%)$ was dissimilar. Explanation of these differing results is likely due to the effect of the different drying regimes. There is evidence that high vacuum drying produces a size decrease of individual colloids by about $50 \%,{ }^{12}$ while images here indicate that high vacuum drying may also cause aggregation, increasing colloid sizes dramatically.

The results show that there may be serious implications if one of the techniques was used on its own to assess the suitability of CFF for the size fractionation of aquatic colloids and particles. Indeed, ESEM suggested that the fractionation was relatively poor and that the retentate was largely contaminated by small colloids, whereas SEM suggested that the fractionation had been adequate. The literature includes evidence, from electron and atomic force microscopy data, that smaller colloids (including those smaller than $50 \mathrm{~nm}$ ) are generally present in large amounts in all CFF-generated fractions including the retentates and that $\mathrm{CFF}$ fractionation is not fully quantitative and not based on size alone. ${ }^{12,38}$ In this study, SEM failed to identify the presence of substantial amounts of small colloids in the retentate, presumably due to their aggregation during the drying process. This suggests that ESEM is more appropriate for the determination of particle size distribution than SEM and provide sets of more realistic data.

\section{Advantages and technical constraints: SEM vs. ESEM}

A few technical constraints must be considered in order to produce good quality images using ESEM in comparison to 
SEM. First, the focussing process is challenging owing to partial coverage of the samples by free water, which makes the image acquisition more difficult. In addition, in wet environments, the water surrounding the samples and the samples themselves may be displaced by the electron beam. These two limitations render the whole process of recording images much slower, with the consequence of potentially damaging the fragile nature of the wet samples with the electron beam in an irreversible manner. ${ }^{39}$ However, electron beam damage can be minimised by using appropriate experimental conditions ${ }^{16}$ and by ensuring minimal exposure of the sample to the beam. Another source of difficulty is the need to lower the relative humidity in the microscope chamber to fully visualise the sample and reduce its coverage by water. This may introduce artefacts such as aggregation ${ }^{29}$ and might hinder accurate recording of specific fragile components of natural waters. However, these constraints are more than compensated for by the fact that (i) ESEM permits live recording of any changes in the morphologies and conformation of the samples during the reduction in relative humidity, which helps to identify the occurrence of artefacts, (ii) ESEM allows the observation of wet samples in their native state, (iii) the high vacuum requirements of SEM and the drying of the wet specimens prior to imaging cause drastic changes in colloid morphology, and (iv) ESEM, in contrast to SEM, does not require coating of samples. ${ }^{29,40,41}$ Indeed the last two processing steps (iii) and (iv) in ESEM help to minimise the introduction of artefacts and the damage of finer features of the specimens. However, SEM has advantages in that it provides much clearer images at lower resolution.

\section{Conclusions}

SEM and ESEM have been used together in order to visualise estuarine colloids before and after fractionation by CFF. Based on the results and our a priori knowledge of the mechanisms by which the two microscopy techniques work, we suggest that ESEM is the preferred method for imaging these colloids. ESEM results in a more representative understanding of colloidal structure because of the absence of staining and drying artefacts inherent to SEM. The differences in the images suggest that aggregation and dehydration has occurred extensively in the SEM images. However, combination with SEM (and other methods) allows more information to be extracted on the nature of these polydisperse materials and this approach, using a number of techniques, is to be recommended.

\section{Acknowledgements}

This research was financially supported by the Natural Environment Research Council (NER/B/5/2002/00544 and NER/A/ S/2000/01112).

\section{References}

1 J. R. Lead, W. Davison, J. Hamilton-Taylor and J. Buffle, Aquat. Geochem., 1997, 3, 213.

2 J. R. Lead, J. Hamilton-Taylor, W. Davison and M. Harper, Geochim. Cosmochim. Acta, 1999, 63, 1661.

3 K. J. Wilkinson, E. Balnois, G. G. Leppard and J. Buffle, Colloids Surf., A: Physicochem. Eng. Aspects, 1999, 155, 287.
4 K. J. Wilkinson and J. Buffle, in Physicochemical Kinetics and Transport at Biofaces, ed. H. P. van Leeuwen and W. Koster, Wiley, New York, 2004.

5 W. Stumm, Colloids Surf., A: Physicochem. Eng. Aspects, 1993, 73, 1 .

6 G. G. Leppard, in Environmental Particles, ed. J. Buffle and H. P. van Leeuwen, Lewis Publishers, Boca Raton, FL, 1992, Vol. 1, pp. 231-290.

7 J. R. Lead, E. Balnois, M. Hosse, R. Menghetti and K. J. Wilkinson, Environ. Int., 1999b, 25, 245.

8 J. R. Lead, K. J. Wilkinson, E. Balnois, B. J. Cutak, C. K. Larive, S. Assemi and R. Beckett, Environ. Sci. Technol., 2000, 34, 3508.

9 P. H. Santschi, E. Balnois, K. J. Wilkinson, J. W. Zhang, J. Buffle and L. D. Guo, Limnol. Oceanogr., 1998, 43, 896.

10 E. Balnois and K. J. Wilkinson, Colloids Surf., A: Physicochem. Eng. Aspects, 2002, 207, 222.

11 M. Plaschke, J. Romer and J. I. Kim, Environ. Sci. Technol., 2002, 36, 4483.

12 F. J. Doucet, L. Maguire and J. R. Lead, Anal. Chim. Acta, 2004, 522, 59.

13 D. Mavrocordatos and D. Perret, J. Microsc. (Oxford), 1998, $191,83$.

14 C. Mondi, K. Leifer, D. Mavrocordatos and D. Perret, J. Microsc. (Oxford), 2002, 207, 180.

15 G. D. Danilatos, Adv. Electron. Electron. Phys., 1988, 71, 109.

16 J. A. Callow, M. P. Osborne, M. E. Callow, F. Baker and A. M. Donald, Colloids Surf., B: Biointerfaces, 2003, 27, 315.

17 R. Ray, B. Little, P. Wagner and K. Hart, Scanning, 1997, 19, 98.

18 M. G. Darkin, C. Gilpin, J. B. Williams and C. M. Sangha, Scanning, 2001, 23, 346.

19 C. Habold, S. Dunel-Erb, C. Chevalier, L. Laurent, Y. Le Maho and J. F. Lignot, Micron, 2003, 34, 373.

20 P. J. R. Uwins, Mater. Forum, 1994, 18, 51.

21 D. M. Lavoie, B. J. Little, R. I. Ray, R. H. Bennett, M. W. Lambert, V. Asper and R. J. Baerwald, J. Microsc. (Oxford), 1995, 178, 101 .

22 P. Bruckel, P. Lours, P. Lamesle and B. Pieraggi, Mater. High Temp., 2003, 20, 551.

23 R. Rizzieri, F. S. Baker and A. M. Donald, Polymer, 2003, 44, 5927.

24 I. B. Beech, C. W. S. Cheung, D. B. Johnson and J. R. Smith, Biofouling, 1996, 10, 65.

25 D. G. Allison, B. Ruiz, C. SanJose, A. Jaspe and P. Gilbert, FEMS Microbiol. Lett., 1998, 167, 179.

26 S. Douglas and D. D. Douglas, Geomicrobiol. J., 2000, 17, 275.

27 J. M. Manero, F. J. Gil, E. Padros and J. A. Planell, Microsc. Res. Tech., 2003, 61, 469.

28 H. E. Nuttall and R. Kale, Microsc. Res. Tech., 1993, 25, 439.

29 P. S. Redwood, J. R. Lead, R. M. Harrison and S. Stoll, Environ. Sci. Technol., 2005, in press.

30 G. D. Danilatos, Micron, 1983, 14, 307.

31 R. E. Cameron, USA Microsc. Anal., 1994, May, 17.

32 A. M. Donald, C. He, C. P. Royall, M. Sferrazza, N. A. Stelmashenko and B. L. Thiel, Colloids Surf., A: Physicochem. Eng. Aspects, 2000, 174, 37.

33 A. M. Donald, Curr. Opin. Colloid Interface Sci., 1998, 3, 143.

34 A. M. Donald, Nat. Mater. (London), 2003, 2, 511.

35 C. Kennedy, D. Scott Smith and L. A. Warren, Geochim. Cosmochim. Acta, 2004, 68, 443.

36 J. R. Lead, J. Hamilton-Taylor, N. Hesketh, M. N. Jones, A. E. Wilkinson and E. Tipping, Anal. Chim. Acta, 1994, 294, 319.

37 P. A. Maurice and A. Namjesnik-Dejanovic, Environ. Sci. Technol., 1999, 33, 1538.

38 L. Guo, L. S. Wen, D. Tang and P. H. Santschi, Mar. Chem., 2000, 69, 75.

39 C. P. Royall, B. L. Thiel and A. M. Donald, J. Microsc. (Oxford), 2001, 204, 185 .

40 J. J. Bozzola and L. D. Russell, Electron microscopy: principles and techniques for biologists., ed. M. A. Sudbury, Jones and Bartlett Publishers, Sudbury, 2nd edn., 1998.

41 M. Hayat, Principles and techniques of electron microscopy: biological applications, Cambridge University Press, Cambridge, 4th edn., 2000. 